\title{
Bearing health assessment based on chaotic characteristics
}

\author{
Chen $\mathrm{Lu}^{\mathrm{a}, \mathrm{b}, *}$, Qian Sun ${ }^{\mathrm{a}}$, Laifa Tao ${ }^{\mathrm{a}}$, Hongmei Liu ${ }^{\mathrm{a}}$ and Chuan Lu ${ }^{\mathrm{a}, \mathrm{b}}$ \\ ${ }^{a}$ School of Reliability and Systems Engineering, Beihang University, Beijing, China \\ ${ }^{\mathrm{b}}$ Science and Technology Laboratory on Reliability and Environmental Engineering, Beijing, China
}

Received 20 October 2012

Revised N/A

Accepted 7 December 2012

\begin{abstract}
Vibration signals extracted from rotating parts of machinery carry a lot of useful information about the condition of operating machine. Due to the strong non-linear, complex and non-stationary characteristics of vibration signals from working bearings, an accurate and reliable health assessment method for bearing is necessary. This paper proposes to utilize the selected chaotic characteristics of vibration signal for health assessment of a bearing by using self-organizing map (SOM). Both Grassberger-Procaccia algorithm and Takens' theory are employed to calculate the characteristic vector which includes three chaotic characteristics, such as correlation dimension, largest Lyapunov exponent and Kolmogorov entropy. After that, SOM is used to map the three corresponding characteristics into a confidence value (CV) which represents the health state of the bearing. Finally, a case study based on vibration datasets of a group of testing bearings was conducted to demonstrate that the proposed method can reliably assess the health state of bearing.
\end{abstract}

Keywords: Health assessment, rolling bearing, feature extraction, chaotic characteristics, self-organizing map

\section{Introduction}

Bearings are one of the most important components of rotating machineries, and faults arising in these systems are often due to damages and failures in the elements of bearings. A faulty bearing could result in serious damage if defects occur under the operation condition. Health assessment can help prevent catastrophic failures in critical rotating machinery by determining optimal maintenance schedules and thus avoiding unscheduled downtime and machine failure costs. Therefore, accurate and timely assessment of the bearing health is crucial to prevent the system from malfunction that could cause damage or entire system halt.

A variety of analysis methods exist for health assessment of bearings including: vibration analysis, oil analysis, infrared thermography and motor current signature analysis. Among all of these methods, vibration analysis by using such as frequency domain technique, time domain technique, and time-frequency domain technique have been widely employed to detect bearing failures [1,2]. However, most parts of rotating machinery often contain complex, non-stationary, noisy and nonlinear characteristics. Such characteristics may range from quasi-periodic to completely irregular behaviors. Nevertheless, especially as a machine fails, the vibration characteristics of the machine are changing.

${ }^{*}$ Corresponding author: Chen Lu, Ph.D., School of Reliability and Systems Engineering, Beihang University, Beijing 100191, China. Tel.: +861082316573; E-mail: luchen@buaa.edu.cn. 
It is worth noting that the most important step for implementing the bearing heath assessment process is to find out an appropriate feature set to accurately represent the changes of bearing vibration signals as the health condition changes. However, the works presented in [3] showed that, some frequency domain techniques such as the FFT-based methods are not quite suitable for non-stationary signal analysis since the inherent information of nonstationary signals might not be revealed and the analysis of non-stationary signals requires specific techniques [4]. High frequency resonance analysis (also known as envelope analysis) [5], bicoherence analysis [6] and other frequency domain methods have been used for the identification of these major frequency components in the vibration spectrum for detecting localized defects. In recent years, advanced time-frequency techniques such as, the wavelet transform [7,8] and EMD [9] have also been employed in machinery vibration analysis. However, few studies have focused on bearing health assessment based on nonlinear dynamical analysis.

Nevertheless, in recent time, new approaches based on fractal and deterministic chaos analyses have been adopted to analyze the complex dynamic behavior of nonlinear systems. The dimensional exponent (an approximation of the correlation dimension) derived from the partial correlation integral algorithm was used to diagnose bearings heath condition, as depicted in [10]. The experimental results in [11] showed that the largest Lyapunov exponents of bearing vibration signal were different under different health states of a rolling bearing, and therefore, it can be used as the characteristic for bearing diagnosis. The study in [12] presented a new approach to rotating machine health monitoring based on the Approximate Entropy, and it is actually a statistical measure that quantizes the regularity of a time series, such as vibration signals measured from an electrical motor or a rolling bearing. The studies mentioned above have showed more effective results than traditional vibration-based analysis approaches, regarding the fault diagnosis of rotating machinery. Thus, the non-linear behavior analyses on rotating machinery have received more attentions, due to the presence of instantaneous variations in friction, damping, or loading conditions. Accordingly, some new emerging techniques for non-linear characteristics estimation provide a good alternative to extract defectrelated features, which are hidden in the vibration signals and will be changing, especially as a machine fails.

Bearings as non-linear systems, their vibration signals usually exhibit non-stationary phenomenon with chaotic characteristics. Different chaotic characteristics, e.g., largest Lyapunov exponent, Kolmogorov entropy, and correlation dimension, can represent different nonlinear features of the analyzed vibration signal, and reflect the health state of a bearing from different aspects, respectively. Correlation dimension defines the active degrees of freedom or the complexity of a system operating in nonlinear regime on the attractor [13]. The spectrum of Lyapunov exponents captures the dynamical properties of the system orbiting within the attractor [14]. Largest Lyapunov exponent provides an estimate of the mean exponential divergence or convergence of nearby trajectories in phase space, expressing the sensitivity of dependence on initial states. Kolmogorov entropy evaluates the chaotic degree of a system, or the average velocity at which new information is generated by the system, or equivalently, at which current information about the system is lost [15]. To comprehensively represent the health state of a system, some studies have proposed a proper metric model integrating various chaotic characteristics, which is more effective and accurate in quantizing health states. Yu et al. [16] proposed a new method that integrated correlation dimension and Kolmogorov entropy to identify the fault symptoms of aero-engine system. In [17], a literature review discussed related studies on non-stationary and nonlinear characteristics, which indicates that nonlinear dynamical analysis techniques, such as correlation dimension, largest Lyapunov exponents and Kolmogorov entropy, can be used to investigate the nonlinear dynamical behavior and complexity generated by machines over their operating time. In [18], two chaotic characteristics (correlation dimension and exponents and Kolmogorov entropy) extracted from the torque signals of a diesel engine were integrated to identify the fault symptoms in different fault modes. These results of fault diagnosis indicate that the model integrating at least two chaotic characteristics shows higher performance than those only using one characteristic. Therefore, single chaotic characteristic, extracted from vibration signal, is not comprehensive in accurately assessing the health states of a machine or component; alternatively, methods on fault diagnosis and health assessment by effectively integrating all possible chaotic characteristic parameters should be developed for rotating machinery.

It can be seen from the above literatures that chaos analysis methods about the mentioned characteristics have been used in great quantities; however, few works have been conducted on how to properly select and utilize these chaotic characteristics for a satisfactory health assessment, which is just the key premise of bearing health assessment. In this study, the authors calculate the correlation dimension, Kolmogorov entropy and largest Lyapunov exponent of bearing vibration signals under normal and fault states, respectively. The experimental results prove that, the 
bearing vibration signals usually exhibit nonlinear chaotic characteristics even though under normal states; and that once any fault occurs with aggravating shocks, the signal will present enhancement of uncertainty and randomness, still remaining distinct chaotic characteristics.

Based on these existing works, this study proposes a new model for bearing health assessment by integrating appropriate nonlinear chaotic characteristics. First, three selected chaotic characteristics, such as the correlation dimension, largest Lyapunov exponent and Kolmogorov entropy, are extracted from the vibration signal of a rolling bearing to construct the corresponding feature vector. Second, every new feature vector is input to a self-organizing map (SOM) neural network, which is trained only using the dataset acquired under normal states. Then, the Minimum Quantization Error (MQE) can be obtained and normalized into a confidence value, which indicates the health state of the bearing, ranging from 0 to 1 . The experimental results show that there is a change on the chaotic characteristic vector of bearing vibration signals as any fault occurs, and the three chaotic characteristics can be used to effectively monitor the health state of a bearing.

\section{Feature extraction}

In this case, correlation dimension, largest Lyapunov exponent and Kolmogorov entropy are used to investigate the nonlinear dynamic behaviors and chaotic characteristics generated by bearings over their operating life time.

The algorithm discussed in this paper for the computation of the three chaotic parameters is based on the phase space reconstruction of nonlinear system using time-delay embedding scheme, referred to as the Takens reconstruction [4].

We begin with a univariate time series $\{x[i] \mid i=1,2, \ldots n-1, n\}$, where each sample $x[i]$ is a one-dimensional measurement of the nonlinear system being analyzed. To provide a more complete description of the higher dimensional nonlinear system dynamics, the time series $x$ needs to be unfolded into a higher dimensional space $R^{m}$.

The $m$-dimensional embedding vector $X \in R^{m}$ of the time series $x$ is given as bellow.

$$
X(i)=[x(i), x(i+\tau), x(i+2 \tau), \ldots, x(i+(m-1) \tau)]\left(i=1,2, \ldots, n_{m}\right)
$$

Where $n_{m}=n-(m-1) \tau$ is the number of new vectors in the reconstructed space, $m$ and $\tau$ are the embedding parameter denoting the embedding dimension and the time-delay, respectively.

\subsection{Correlation dimension}

The correlation integral and dimension developed by Grassberger and Procaccia [19] are two of the most popular nonlinear techniques in the field of nonlinear time series analysis. Practically, the correlation dimension of a reconstructed space is estimated from the correlation integral $C\left(n_{m}, r, t\right)$, which measures the probability that points on the attractor have pairwise distances less than or equal to the corresponding distance $r$.

The commonly used method for estimating the correlation dimension is the Grassberger-Procaccia algorithm (GPA) [19], in which the correlation integral $C\left(n_{m}, r, t\right)$ of a time series $x$ is defined as

$$
C\left(n_{m}, r, t\right)=\frac{2}{n_{m}\left(n_{m}-1\right)} \sum_{i=1}^{n_{m}} \sum_{j=1}^{n_{m}} H(r-D(i, j))
$$

Where the Heaviside function $H(x)=1$ if $x \geqslant 0,0$ otherwise. And the distribution of distances $H(r-D(i, j))$ specifies the total number of the pairs of embedding vectors $\left(x_{i}, x_{j}\right)$ such that the distance between the embedding vectors $x_{i}$ and $x_{j}$ is less than the specified distance $r$.

The correlation integral $C\left(n_{m}, r, t\right)$ behaves as a power of $\gamma$ for $r$, as Eq. (3)

$$
C\left(n_{m}, r, t\right) \propto r^{\gamma}
$$

Where the exponent $\gamma$ for $r$ defines the correlation dimension $d(m, t)$ and can be calculated by

$$
d(m, t)=\lim _{r \rightarrow 0} \frac{\log C\left(n_{m}, r, t\right)}{\log (r)}
$$

In this study, the correlation dimension $d(m, t)$ is determined from an estimate of the local slope of the log of the correlation integral $C\left(n_{m}, r, t\right)$ versus $\log (r)$ using the estimation scheme proposed in [10]. 


\subsection{Largest Lyapunov exponent}

According to Grebogi's proving, a clearly positive value of the largest Lyapunov exponent provides evidence that the $m$-dimensional dynamic system under investigation is chaotic. Here, the method for calculating the largest Lyapunov exponent is introduced in [14].

Symbol $\|||$ is supposed to denote the distance in $m$-dimensional space, and then the largest Lyapunov exponent $\lambda_{1}$ should satisfy the relation as bellow

$$
\left\|\delta x_{0}\right\| e^{n \lambda_{1}}=\left\|F^{n}\left(x_{0}+\delta x_{0}\right)-F^{n}\left(x_{0}\right)\right\| \approx\left\|J\left(x_{n-1}\right) J\left(x_{n-2}\right) \ldots J\left(x_{0}\right) \delta x_{0}\right\|
$$

Where $\left\|\delta x_{0}\right\|$ is the distance between the pair of points at the initial position, and $n$ denotes the iterations. $J\left(x_{0}\right)$ is the Jacobian matrix of the dynamic system which is in the initial position.

In order to get the value of $\lambda_{1}$, we firstly calculate the eigen value of each Jacobian matrix. And then

$$
\lambda_{1}=\lim _{n \rightarrow \infty} \frac{1}{n} \ln \left|d_{n-1} d_{n-2} \cdots d_{1} d_{0}\right|
$$

Where $d_{0} \ldots d_{n-1}$ are eigen values of $J\left(x_{0}\right) \ldots J\left(x_{n-1}\right)$.

Equation (6) shows that largest Lyapunov exponent represents an estimate of the mean exponential divergence or convergence of nearby trajectories in phase space.

\subsection{Kolmogorov entropy}

Suppose that the phase space is divided into several $m$-dimensional units, whose side length and total numbers are $\varepsilon$ and $n$. The system is observed in a very small time interval $\tau$, then $P_{i}$ is defined as the probability that the vector $x(i \tau)$ drops just into the unit $i$. According to the Shanon theory,

$$
K_{n}=-\sum_{i=0}^{n} P_{i} \ln P_{i}
$$

Kolmogorov entropy is defined as the mean rate at which current information about the system is lost [12].

$$
K=\lim _{\tau \rightarrow 0} \lim _{\varepsilon \rightarrow 0} \lim _{n \rightarrow \infty} \frac{1}{n \tau} \sum_{i=1}^{n}\left(K_{i+1}-K_{i}\right)
$$

Equation (8) indicates the similarity among the reconstructed vectors within the time series, when the dimension of the vectors has increased from $i$ to $i+1$. This affects the regularity of the time series being analyzed, consequently, the greater the regularity is, the lower the Kolmogorov entropy value.

The value of Kolmogorov entropy of a regularly dynamic system is 0 , while infinity for random systems. Thus, if a dynamic system exhibits a constant positive value of Kolmogorov entropy, it denotes that the system has chaotic characteristics.

\section{Health assessment method}

The health assessment process based on SOM neural network can be visualized as Fig. 1. It can realize an online health assessment for a bearing, using only the dataset under normal condition. The proposed method has no requirements on the full life cycle of performance degradation dataset.

\subsection{SOM neural network}

Self-organizing map (SOM) network is a kind of competitive artificial neural network that is suitable for data clustering [20]. The SOM network combines an input layer with a competitive layer of processing neurons, which typically organized as a two-dimensional grid. The structure of the SOM network is shown in Fig. 2. As shown in Fig. 2, the SOM network is an array of $M=m \times m$ processing neurons, which maps high dimensional input vectors onto a two-dimensional surface, in which each neuron is represented by one-dimensional weight vector. Neurons of the map are connected to adjacent neurons by a neighborhood relation, which indicates the topology of the map. 


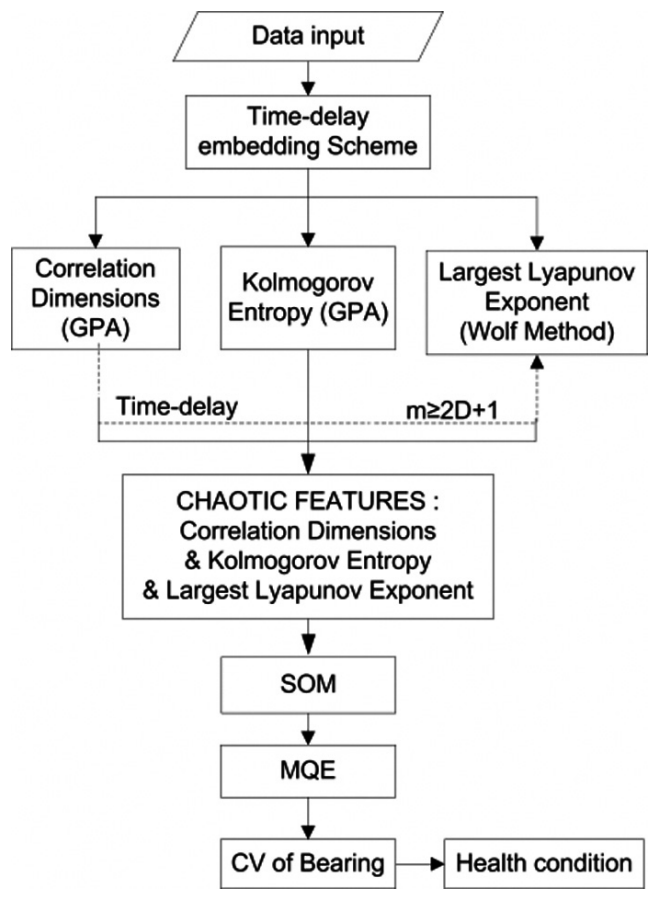

Fig. 1. Process of bearing health assessment.

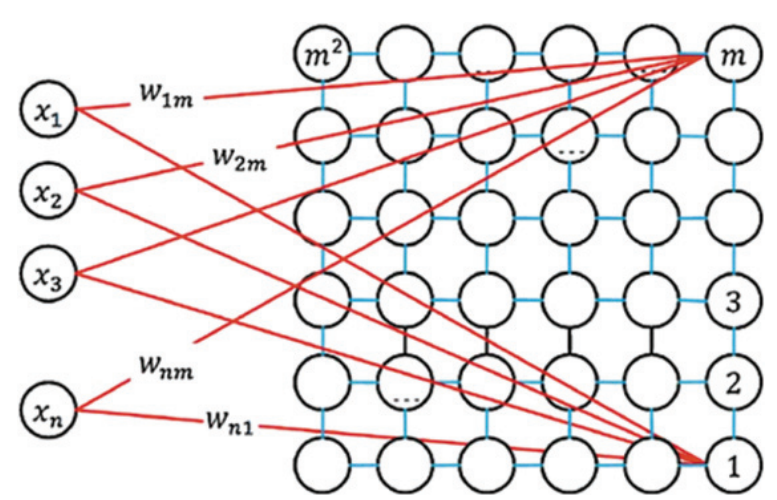

Fig. 2. Structure of SOM network.

The weight vector is enhanced during training procedure by the learning rule:

$$
w_{i}(t+1)=w_{i}(t)+\alpha(t) \cdot h_{B M U, i}(t)\left(x(t)-w_{i}(t)\right)
$$

Where $w_{i}(t)$ is the weight vector; $\alpha(t)$, the learning rate of SOM network; $h_{B M U, i}(t)$, the neighborhood function determined by the distance between the Best Matching Unit (BMU) and the neighbor neurons.

All the neurons are grouped in clusters by their distances through the training process, which form a new kind of topology structure. After that, each group of test data inputted to the well-trained SOM can match with a BMU. Finally, the Minimum Quantization Error (MQE) can be obtained by calculating the Euclidean distance between the BMU and the real-time measurement data.

\subsection{Performance indicator}

According to the principles of SOM, the normal signals with similar features as the training dataset will gather around the normal cluster, which should have a minimum value of MQE. Along with the performance degradation of a bearing, its features from the vibration signal turn to be more and more different from those of the normal condition. Accordingly, the values of MQEs will have an increasing trend over time as the performance of a bearing deteriorates.

In this study, the confidence value $(\mathrm{CV})$, which can be formulated as below, is used to represent the performance of a bearing.

$$
C V=\frac{c}{\sqrt{x}+c}
$$

Where, $c$ is a scale parameter determined by the averaged MQE under normal conditions and the corresponding CV, and $x$ is just the MQE generated by using the online input measurement data, which will be normalized into the $\mathrm{CV}$ ranging from 0 to 1 . It can be clearly seen that the $\mathrm{CV}$ is inverse proportional to the $\mathrm{MQE}$, meaning that worse performance generates a higher MQE and a lower CV. 


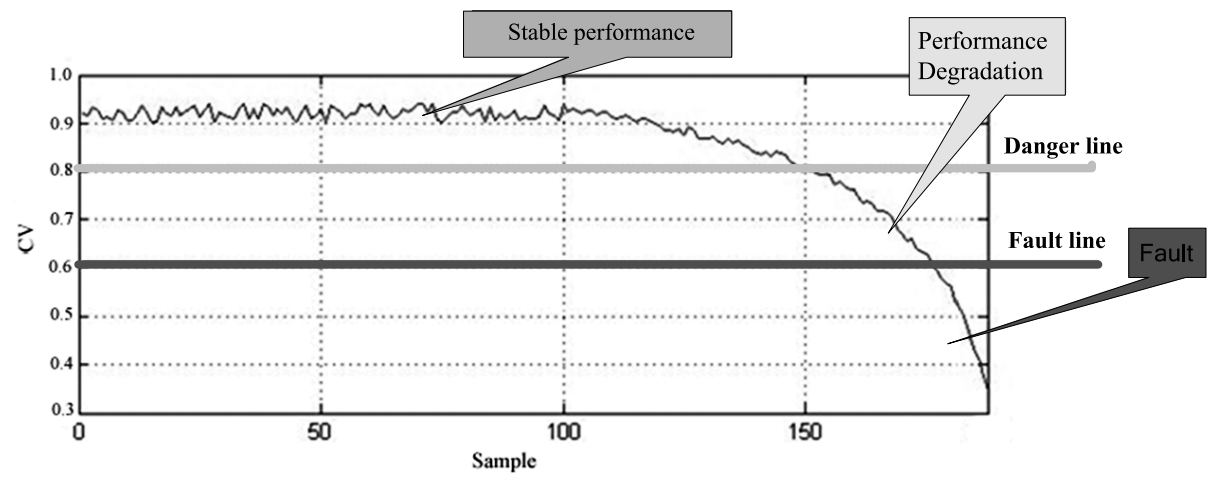

Fig. 3. Different performance degradation states during the life cycle.

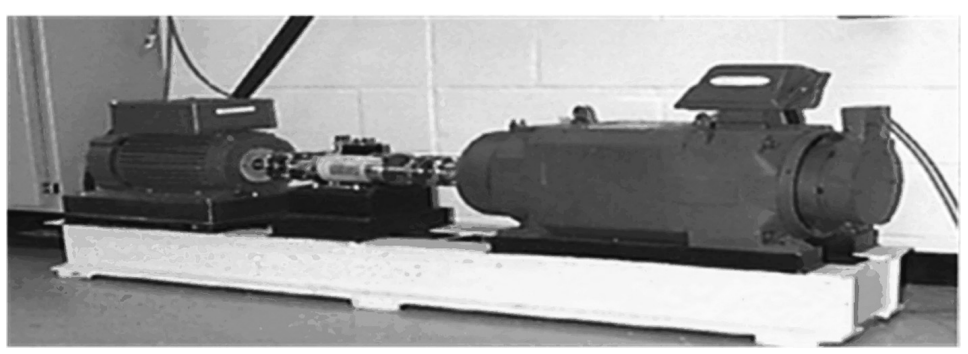

Fig. 4. Experimental setup.

As discussed in [21], instead of failing suddenly, most mechanical system components usually undergo gradual degradation processes up until the time they fail, or scheduled to be replaced. If a component's degrading state is continuously examined, a performance indicator $(\mathrm{CV})$ curve can be obtained as shown in Fig. 3. The CV ranges between 0 (unacceptable) and 1 (normal). Incremental changes in the CV are indicative of a gradual degradation process. No matter what type of degradation pattern the component goes through before it fails, the CV is always initially stable (in the range of 0.9 to 1.0 ). When an incipient fault occurs, the CV will show a decreasing trend. After the initial fault propagates to a severe level, and the component is close to failure, the decrease in the CV becomes more obvious and quickly drops to 0 . Once the $\mathrm{CV}$ drops below the predetermined threshold, we can judge that certain fault occurs.

Traditionally, health assessment is performed by using a single characteristic. A single characteristic, although effective for a specific degradation pattern, cannot achieve accurate health assessment results throughout a component's life cycle. Utilizing multiple characteristics, however, can achieve better assessment accuracy than a single one, although challenges remain in how to dynamically choose a group of optimal characteristics.

\section{Experimental verification}

\subsection{Experimental setup}

The experimental verification of bearing health assessment was conducted by using the acquired data from a designed bearing test-rig, as shown in Fig. 4. The test bearing, a deep groove ball bearing of 6205-2RS JEM SKF, was assembled in the motor. The accelerometer was installed on the magnetic base shell to capture vibration signals of the bearings.

In this experiment, a series of single-point faults injected on three different positions (inner-race, out-race and rolling element), were introduced to the test bearings using electro-discharge machining with the defect diameters of $0.014,0.021$ and 0.028 inches. Vibration data used in this study was acquired at a sampling rate of $12 \mathrm{KHz}$, with a rotating speed of $1772 \mathrm{rpm}$. 

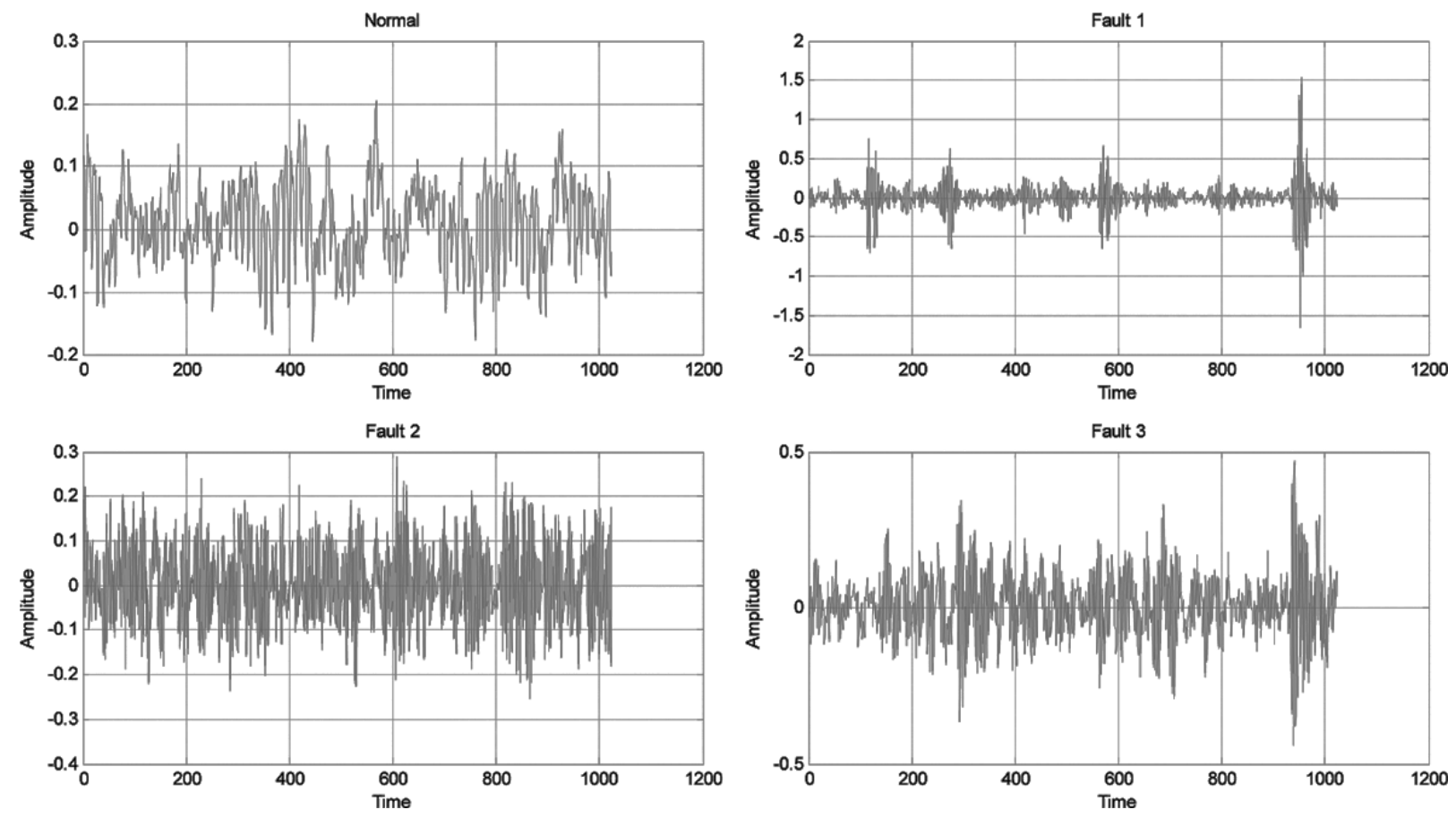

Fig. 5. Representative vibration signals under different bearing conditions in time domain.

\subsection{Chaotic characteristics extraction for training and testing data}

In this experiment, the correlation dimension and Kolmogorov entropy were determined from an estimate of the local slope and intercept of $\log C\left(n_{m}, r, t\right)-\log (r)$ using the estimation scheme proposed in [22], and the largest Lyapunov exponent was calculated by using the Wolf-method [23]. In reference to the Wolf-method, if the exponent estimate converges, it is generally not necessary to evolve entirely for all dataset. In this study, the exponential evolution of the phase space trajectories was established by iteratively calculating for 1024 steps, and then the largest Lyapunov exponent was obtained by an averaging process.

Consequently, each feature vector for the subsequent model training and health assessment consists of the above three chaotic characteristics extracted from the vibration signals of the testing bearings. A certain number of feature vectors generated under normal condition were selected for the training of SOM-based health assessment model, and the remaining groups from normal and three types of fault conditions for the model verification.

For further describing the effectiveness of the selected chaotic characteristics, a testing example was conducted to validate the relationship between the actual health state and each chaotic characteristic. The vibration dataset acquired under normal and three types of fault modes (inner-race/out-race/rolling element fault with the defect diameter of 0.014 inches) was taken for this validation.

The representative time-domain vibration signals under different bearing conditions, e.g., normal, fault 1 (innerrace fault), fault 2 (outer-race fault), and fault 3 (rolling element fault), are shown in Fig. 5. The relationship between $\ln C(r)-\ln (r)$ and bearing health state is shown in Fig. 6. Similarly, it can also be observed that different bearing health states correspond to different Lyapunov exponent spectrum, as shown in Fig. 7, whose mean value is equivalent to the largest Lyapunov exponent. As depicted in Fig. 8, the results of correlation dimension, Kolmogorov entropy and largest Lyapunov exponent taken from 11 groups of normal data and 33 groups of faulty data, were calculated to show the variation of each chaotic characteristic parameter along with the health state, respectively.

It can be observed from results of the above three chaotic characteristics that, compared with the normal condition, the correlation dimension and Kolmogorov entropy from the faulty bearings are distinctly increased, accordingly, which indicates that the nonlinear chaotic characteristic is becoming more obvious. In respect to the result from the largest Lyapunov exponents, it can be used to distinguish the normal bearings from the outer-race/rolling-element 

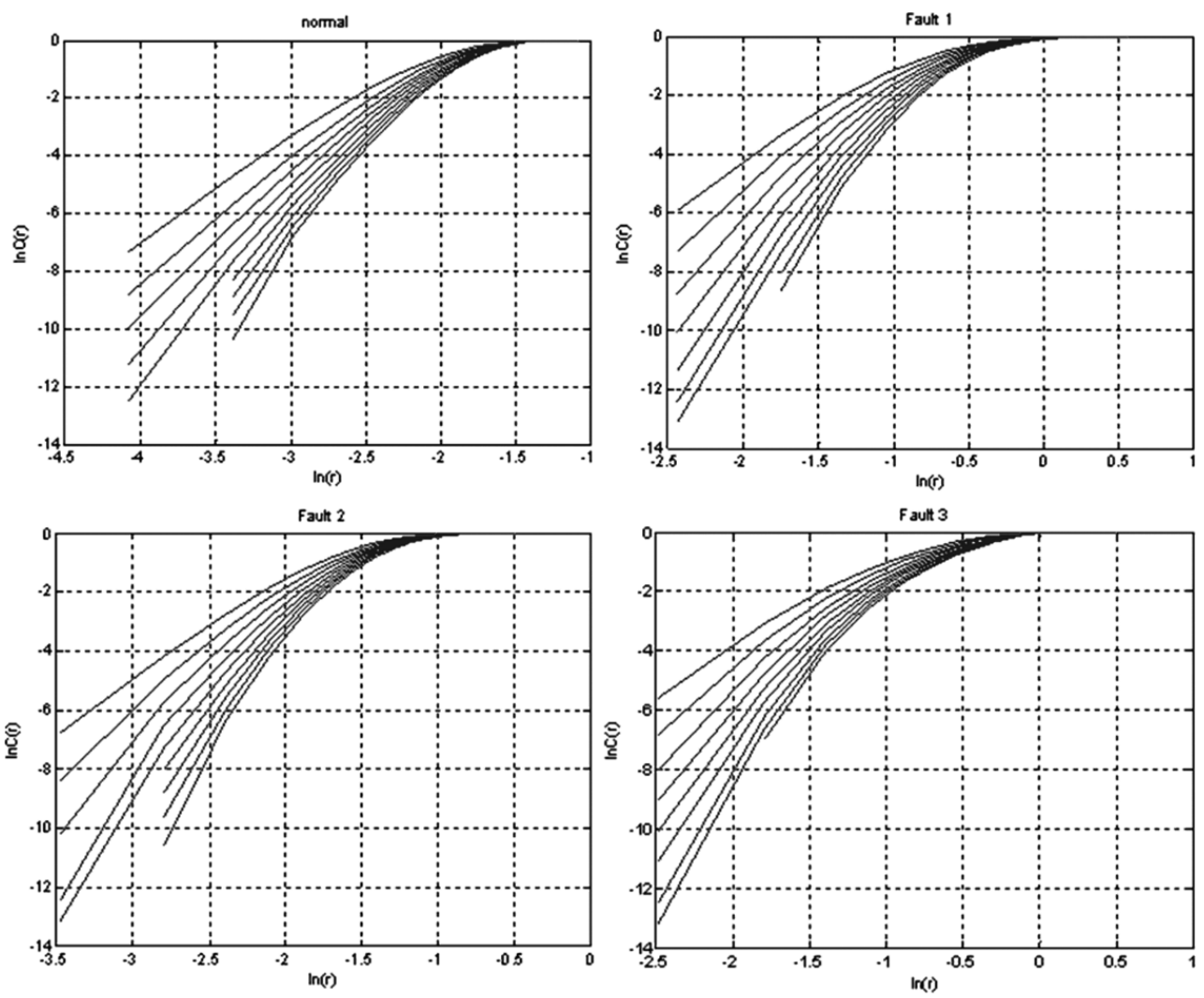

Fig. 6. $\ln C(r)-\ln (r)$ under different health condition.
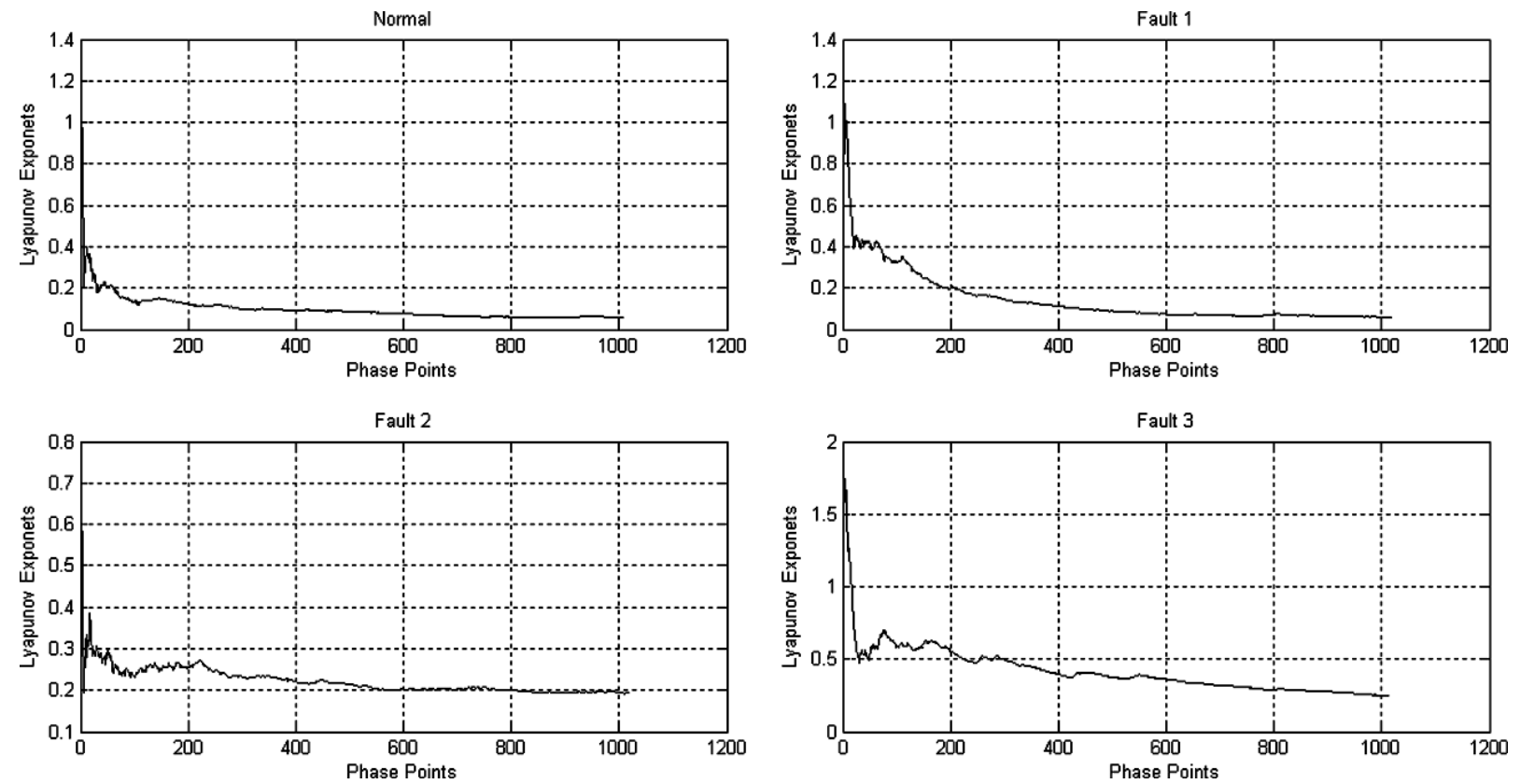

Fig. 7. Lyapunov exponents of vibration signals under different health conditions. 


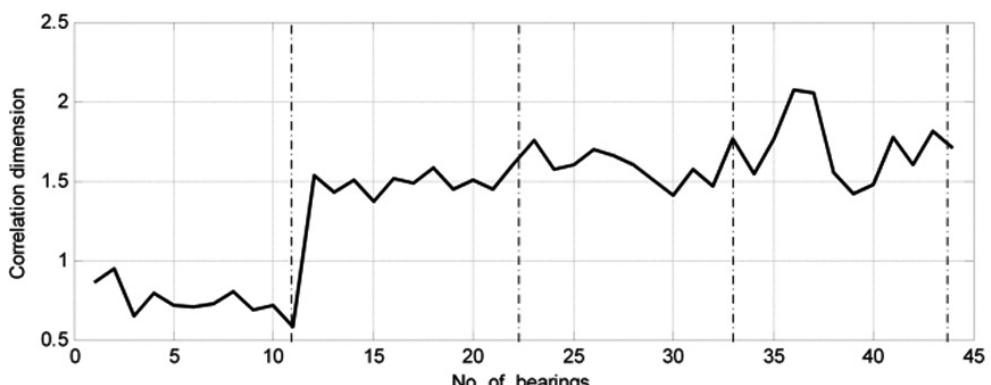

(a)

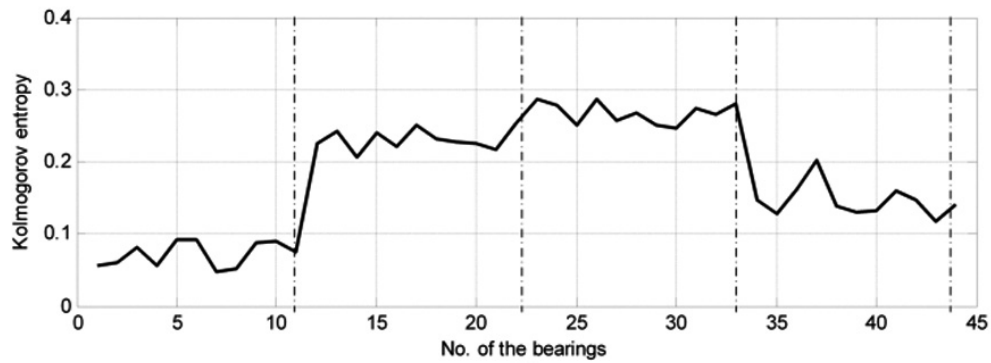

(b)

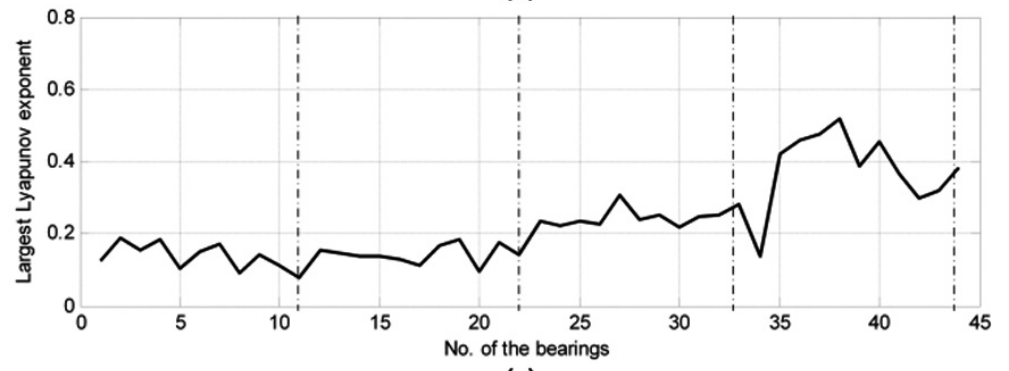

(c)

Fig. 8. Results of the three chaotic characteristic parameters (a) Correlation dimension (b) Kolmogorov entropy (c) Largest Lyapunov exponent.

fault states, even though there is no clear effect on identification of the inner-race fault states. As discussed above, to achieve an accurate health assessment for bearings, these three chaotic characteristics should be extracted and integrated into a new performance indicator.

\subsection{Bearing health assessment}

Then, the chaotic characteristic vectors of 50 groups of normal data were used to train a SOM for establishing the health assessment model. For model verification, 11 groups of normal data and 33 groups of faulty data consisting of three types of fault modes (inner-race/out-race/rolling element fault, each with the defect diameter of 0.014 inches), were taken and sequentially imported into the trained SOM-based health assessment model. The performance assessment results are shown in Fig. 9.

The performance degradation is clearly shown in the two figures. An appropriate threshold should be predetermined to indicate the degradation of bearing health state, and it is set as 0.65 according to previous experience in this study. The CVs of the first 11 samples, collected from the normal data, are highly above the predetermined failure threshold, which shows a stable health state. However, the other CVs from the faulty data are below the threshold. Thus, the health assessment can be achieved, and it is also distinctly proved that the assessment result based on MQE is well consistent with that using CV.

Besides, for further verification, 11 groups of normal data and 33 groups of inner-race fault data, consisting of three types of fault degrees e.g., defect diameter of 0.014 inches, defect diameter of 0.021 inches and defect diameter 


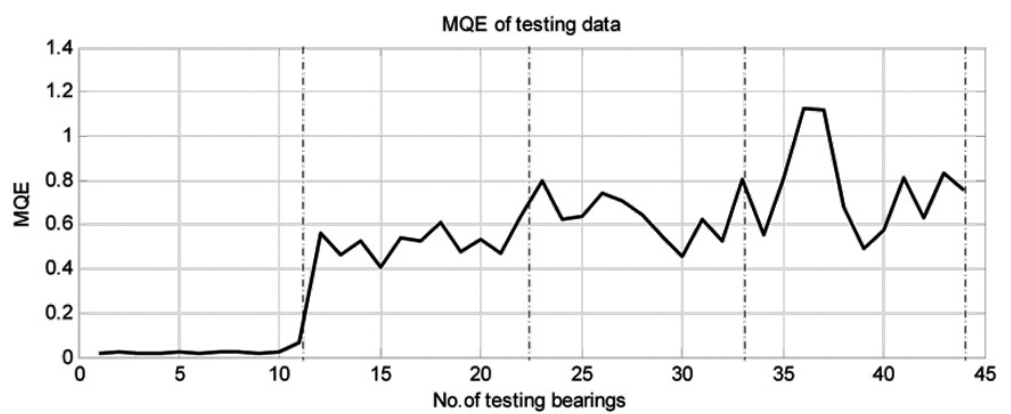

(a)

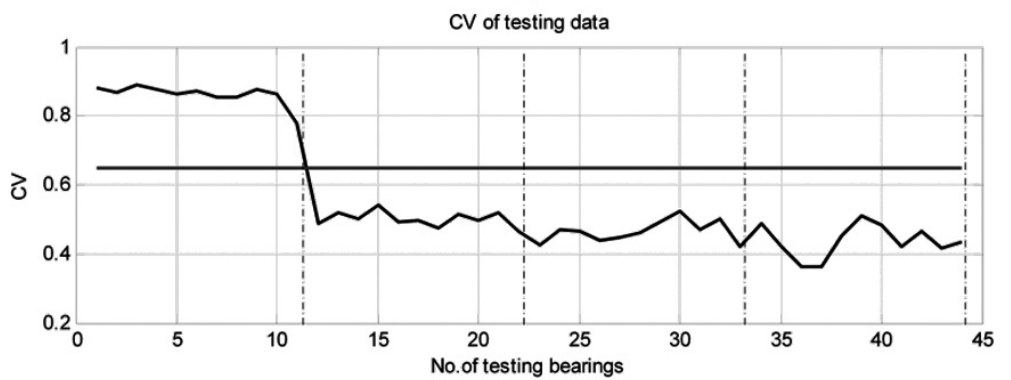

(b)

Fig. 9. Bearing health assessment of different fault modes with a defect diameter of 0.014 inches (a) Using MQE (b) Using CV.

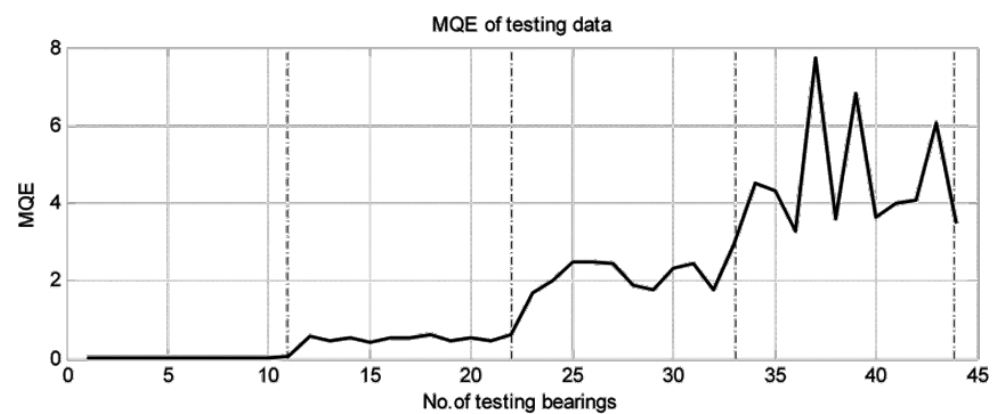

(a)

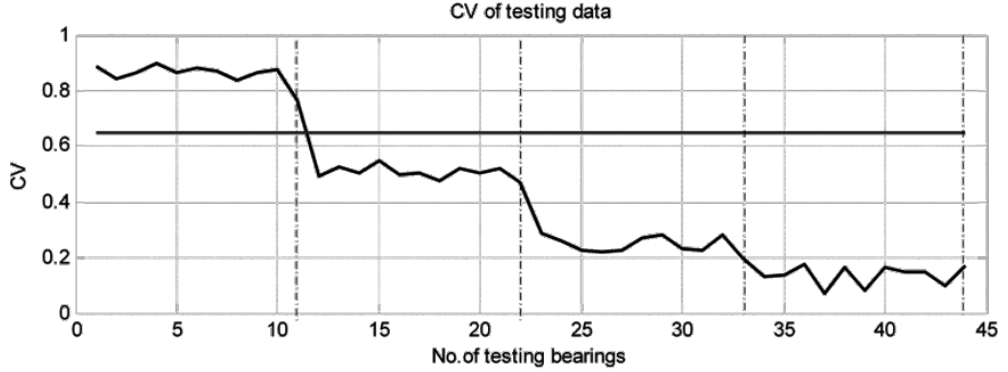

(b)

Fig. 10. Bearing health assessment of different fault degrees (inner-race fault), (a) Using MQE (b) Using CV.

of 0.028 inches, were selected and sequentially imported into the trained SOM-based health assessment model. The performance assessment results are shown in Fig. 10.

This experimental results show that there is a descending trend of the CVs as the fault degrees of the testing bearings are increased from 0.014 to 0.028 inches, which is consistent with the actual health state of the testing bearing sequence (the first 11 bearings are normal, and the left 33 bearings in fault state). It also implies that 
the chaotic characteristics of the fault signals from the faulty bearings distinctly deviate from those under normal conditions. The predetermined failure threshold (set as 0.65 ) can effectively distinguish between a healthy and defect bearing; the $\mathrm{CV}$ can be used to monitor the health of a bearing.

In summary, it have been seen that the corresponding chaotic characteristics extracted from the bearing vibration data are becoming more obvious as the bearings deteriorate. Moreover, the proposed method has no dependencies on failure samples/performance degradation data for its model establishment, and could be more pragmatic for practical applications.

\section{Conclusions}

Considering the strong non-linear and complexity characteristics of vibration signals from running bearings, this paper has outlined the definitions of three typical chaotic characteristics such as the correlation dimension, largest Lyapunov exponent and Kolmogorov entropy, which have the highest potential to describe the various health states. These chaotic characteristics were extracted by using both Grassberger-Procaccia algorithm and Takens' theory, then was fed as input to SOM neural network which map the three corresponding characteristics into a confidence value $(\mathrm{CV})$ representing the health state of the bearing.

From the experimental results given in this paper, the feasibility and efficiency of this method has been verified, that if a proper threshold of the $\mathrm{CV}$ is chosen, a suitable early warning of an impending bearing failure can be realized.

Generally, the attractive advantage of the proposed health assessment model is that: it requires only the normal data for model training and establishment, without the need of full life cycle of degradation data.

Further research must be carried out on this health assessment model: first, only the case in which the normal and three fault types of vibration dataset have been analyzed in depth in this paper. Therefore, it seems of interest to further analyze the case where we plan to sequentially acquire on-line degradation measurements that are representative of the entire bearing life cycle and thus further validate the proposed models. Second, the question of how to reasonably scale the health level for accurate expression of health state (health, warning, sub-health, danger, and failure) still remains. Most importantly, there may be further possible improvements or optimizations for the health assessment model through the integration of information regarding the chaotic characteristics and statistical indicators in time-frequency domain. Third, future work will also be considered to further validate the availability of this method in health assessment of other parts of rotating machinery such as gear box, pump, etc.

\section{Acknowledgments}

This research is supported by the National Natural Science Foundation of China (Grant No. 61074083, 50705005, and 51105019) as well as the Technology Foundation Program of National Defence (Grant No. Z132010B004). The authors are very grateful to the reviewers and the editor for their valuable comments and suggestions.

\section{References}

[1] L.L. Jiang, Y.L. Liu, X.J. Li and A.H. Chen, Degradation assessment and fault diagnosis for roller bearing based on AR model and fuzzy cluster analysis, Shock and Vibration 18 (2011), 127-137.

[2] J.S. Cheng, D.J. Yu, J.S. Tang and Y. Yang, Application of SVM and SVD technique based on EMD to the fault diagnosis of the rotating machinery, Shock and Vibration 16 (2009), 89-98.

[3] H.V. Liew and T.C. Lim, Analysis of time-varying rolling element bearing characteristics, Journal of Sound and Vibration 283 (2005), 1163-1179.

[4] T.R. Kurfess, S. Billington and S. Liang, Advanced diagnostic and prognostic techniques for rolling element bearings, in: Advanced Manufacturing, Springer, London, 2006, pp. 137-165.

[5] P.D. McFadden and J.D. Smith, Vibration monitoring of rolling element bearings by high frequency resonance technique - a review, Tribology International 17 (1984), 3-10.

[6] C.J. Li, J. Ma and B. Hwang, Bearing localized defect detection by bicoherence analysis of vibrations, Journal of Engineering for Industry 117 (1995), 625-629. 
[7] T. Yu and Q.K. Han, Time frequency features of rotor systems with slowly varying mass, Shock and Vibration 18 (2011), 29-44.

[8] C.H. Chen, R.J. Shyu and C.K. Ma, A new fault diagnosis method of rotating machinery, Shock and Vibration 15 (2008), 585-598.

[9] J.S. Cheng, D.J. Yu, J.S. Tang and Y. Yang, Application of SVM and SVD technique based on EMD to the fault diagnosis of the rotating machinery, Shock and Vibration 16 (2009), 89-98.

[10] S. Janjarasjitt, H. Ocak and K.A. Loparo, Bearing condition diagnosis and prognosis using applied nonlinear dynamical analysis of machine vibration signal, Journal of Sound and Vibration 317 (2008), 112-126.

[11] L.Y. Wang, H. Wang, K.L. Zhang and W.G. Zhao, Application of Lyapunov exponents to fault diagnosis of rolling bearing, Noise and Vibration Control 5 (2007), 104-105.

[12] R.Q. Yan and R.X. Gao, Approximate Entropy as a diagnostic tool for machine health monitoring, Mechanical Systems and Signal Processing 21 (2007), 824-839.

[13] M.C. Serban and D.M. Dobrea, Complexity analysis of the visual-motor cross-modalities using the correlation dimension parameter, Studies in Health Technology and Informatics 116 (2005), 465-470.

[14] D. Yilmaz and N.F. Güler, Analysis of the Doppler signals using largest Lyapunov exponent and correlation dimension in healthy and stenosed internal carotid artery patients, Digital Signal Processing 20 (2010), 401-409.

[15] F.D. Marques, E.M. Belo, V.A. Oliveira, J.R. Rosolen and A.R. Simoni, On the investigation of state space reconstruction of nonlinear aeroelastic response time series, Shock and Vibration 13 (2006), 393-407.

[16] B.Yu, Y.H. Li and P. Zhang, Application of correlation dimension and Kolmogorov entropy in aeroengine fault diagnosis, Journal of Aerospace Power 21 (2006), 219-224.

[17] H. Kantz and T. Schreiber, Nonlinear Time Series Analysis [M], Cambridge University Press, the United Kingdom, 2001 , pp. 3-10.

[18] J.M. Lu, Fault diagnosis of diesel engines based on chaos method, Diesel Engine 27 (2005), 8-13.

[19] P. Grassberger and I. Procaccia, Characterization of strange attractors, Physical Review Letters 50 (1983), 346-349.

[20] K.P. Kumar, K.V.N.S. Rao, K.R. Krishna and B. Theja, Neural network based vibration analysis with novelty in data detection for a large steam turbine, Shock and Vibration 19 (2012), 25-35.

[21] L.X. Liao, An Adaptive Modeling for Robust Prognostics on a Reconfigurable Platform, Doctoral dissertation, University of Cincinnati, 2010 .

[22] G.B. Zhao, Y.F. Shi, W.F. Duan and H.R. Yu, Calculating correlation demission and Kolmogorov entropy from chaotic time series, Computational Physics 16 (1999), 309-315.

[23] A. Wolf, J.B. Swift, H.L. Swinney and J.A. Vastano, Determining Lyapunov exponents from a time series, Physica D 16 (1985), $285-317$. 

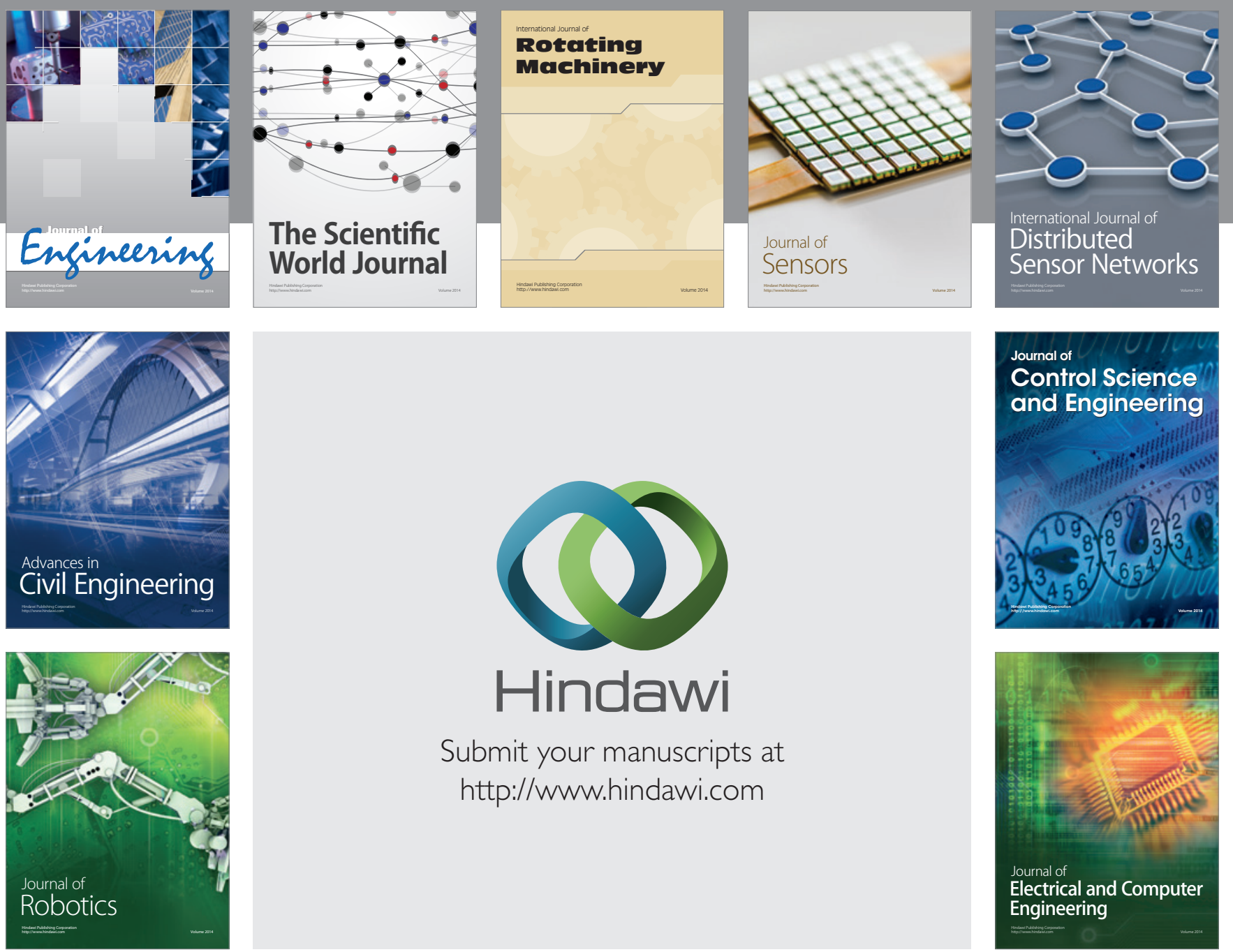

Submit your manuscripts at

http://www.hindawi.com
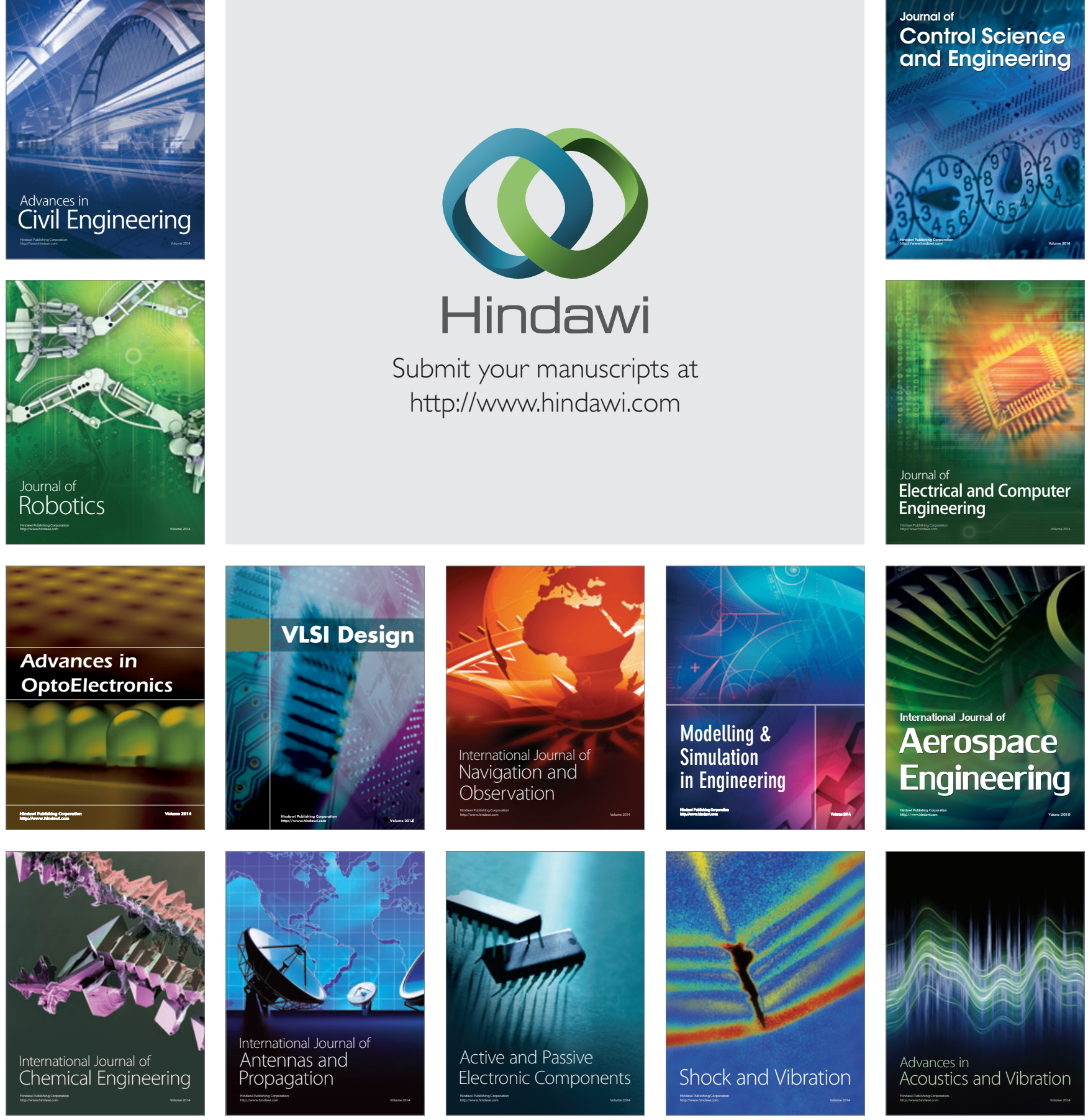